\section{(1)}

CrossMark

\title{
Pirfenidone in the kaleidoscope: reflecting mechanisms through different angles
}

\author{
Argyris Tzouvelekis ${ }^{1,2}$ and Paul J. Wolters ${ }^{3}$
}

Affiliations: ${ }^{1} 1$ st Academic Dept of Respiratory Medicine, Medical School, National and Kapodistrian University of Athens, Hospital for Diseases of the Chest, "Sotiria", Athens, Greece. "Biomedical Sciences Research Center, "Alexander Fleming", Division of Immunology, Athens, Greece. ${ }^{3}$ Division of Pulmonary, Critical Care, Allergy and Sleep Medicine, Dept of Medicine, University of California, San Francisco, CA, USA.

Correspondence: Argyris Tzouvelekis, 1st Academic Dept of Respiratory Medicine, National and Kapodistrian University of Athens, Hospital for Diseases of the Chest, "Sotiria", Messogion 152, 11527, Athens, Greece. E-mail: argyrios.tzouvelekisafleming.gr

@ERSpublications

A multilayer reductionist approach to validate key findings from global gene expression analysis, and identify novel biomarkers and possible therapeutic targets for a drug that advanced to the clinical setting with a relatively unknown mechanism of action http://ow.ly/GUf630myAI0

Cite this article as: Tzouvelekis A, Wolters PJ. Pirfenidone in the kaleidoscope: reflecting mechanisms through different angles. Eur Respir J 2018; 52: 1802046 [https://doi.org/10.1183/13993003.02046-2018].

Pulmonary fibrosis represents the end stage of a diverse group of heterogeneous interstitial lung diseases that are characterised by the destruction of the pulmonary parenchyma, deposition of extracellular matrix (ECM), and dramatic changes in the phenotype of both fibroblasts and alveolar epithelial cells [1, 2]. More than 200 causes of pulmonary fibrosis have been identified, of which the most common form is idiopathic pulmonary fibrosis (IPF) [3]. IPF affects $\sim 5$ million people worldwide and is responsible for more than 40000 deaths per year in the USA, similar in number to breast cancer [4], establishing it as the non-cancer lung disease with the gravest prognosis [3].

These bleak epidemiological statistics will change as new therapies are being developed that limit the progression of IPF. Pirfenidone was the first drug to be approved for the treatment of IPF [5]. Pirfenidone is a pleiotropic molecule with antifibrotic, anti-inflammatory and antioxidant properties. It has been shown to block major, fibrosis-relevant signal transduction pathways, including those of transforming growth factor (TGF)- $\beta 1$, fibroblast growth factor and interleukin (IL)-1 $\beta$, in vivo in mice [6]. In the clinical setting, five randomised controlled trials and several real-life clinical studies [7-11] have reported the beneficial effects of pirfenidone in patients with IPF. In particular, it has been shown to slow the annual rate of forced vital capacity decline, reduce the risk of death at 1 year by almost $50 \%[9,10]$ and prevent respiratory-related hospitalisations, suggesting a therapeutic impact on acute exacerbations [12].

Despite clinical studies confirming clinical efficacy of pirfenidone, mechanistic insights explaining the exact role pirfenidone has in slowing IPF progression in humans are limited. A recent study by NEIGHBORS et al. [13] measured 12 biomarkers (including CCL13, CCL17, CCL18, CXCL13, CXCL14, cartilage oligomeric matrix protein, IL-13, matrix metalloprotease (MMP)3, MMP7, osteopontin, periostin and YKL40) reported to be prognostically or mechanistically relevant to IPF in patients enrolled in the CAPACITY [13] or ASCEND trials [9]. Although circulating levels for several biomarkers were found to be prognostic for progression outcomes, pirfenidone did not have a pharmacodynamic effect on circulating 
levels of any of the biomarkers [13], suggesting it did not impact pathways driving expression of these biomarkers in IPF patients. ZHANG et al. [14] analysed explanted lungs from IPF patients treated with pirfenidone and showed that pirfenidone therapy did not impact expression of senescence markers or levels of phosphorylated SMAD but was associated with less lung injury quantified histopathologically. Clearly, more work needs to be done to define the cellular and molecular pathways favourably impacted by pirfenidone therapy. Identifying these pathways will advance understanding of the pathobiology of IPF and enable development of novel therapies.

Towards this end, in this issue of the European Respiratory Journal, KwAPISZEWSKA et al. [15] used global transcriptome profiling in lung homogenate samples as well as freshly isolated human lung fibroblasts from IPF patients treated with or without pirfenidone to generate data that yielded new mechanistic data on the pharmacological effects of pirfenidone. Total lung homogenate profiling identified significant changes in inflammatory processes and cell-cell contact while the most significant perturbed pathways in fibroblasts were related to metabolic reprogramming, growth and cell division. Genes regulated in both systems primarily belonged to the ECM, although in fibroblasts, the most upregulated genes were contained within the "cytosol" and "nucleus", and in lung homogenates, were overrepresented in "immune responses". Ex vivo effects of pirfenidone were complemented with in vitro experiments on IPF fibroblasts cultured, after several passages, in the presence or absence of pirfenidone. Comparative expression profiling between in vitro IPF fibroblasts and ex vivo fibroblasts isolated from treated and untreated IPF patients revealed overlapping pirfenidone-regulated gene expression patterns involving TGF- $\beta 1$ signal transduction and lipid metabolism pathways. Identification of CEMIP (cell migration-inducing and hyaluronan-binding protein) as one of the most downregulated genes following pirfenidone treatment, in both compartments, was of particular relevance to lung fibrosis. To this end, a detailed characterisation of CEMIP expression revealed the following novel findings. 1) CEMIP expression was increased in both IPF lungs and lung fibroblasts, and was downregulated following pirfenidone treatment. 2) Immunolocalisation studies revealed that structural cells, including alveolar type II epithelial cells, endothelial cells and myofibroblast foci, were sources of CEMIP expression within the fibrotic lung. 3) Since CEMIP is a secreted protein, the authors investigated circulating CEMIP levels in patients with IPF, finding them to be significantly increased compared to controls. These levels were lower in IPF patients treated with pirfenidone, suggesting a pharmacodynamic effect of the drug. 4) Mapping the CEMIP genomic region revealed the presence of key regulatory transcription factors, such as glioma-associated oncogene homologue (GLI), in close proximity to its promoter region. Functional experiments using GLI inhibitors and pirfenidone resulted in downregulation of CEMIP expression. 5) Loss of function experiments demonstrated CEMIP as a positive regulator of fibroblast homeostasis including proliferation, migration and collagen production. No effects on apoptosis and fibroblast differentiation were observed. 6) Lungs from patients with IPF treated with pirfenidone demonstrated increased hyaluronic acid deposition, suggesting a key role for CEMIP in hyaluronan metabolism.

The present study validates prior impressions that pirfenidone has pleiotropic actions on both the immune system and ECM (table 1) [16-18]. The study also reports, for the first time, that pirfenidone may influence metabolism of hyaluronan, a major component of the ECM that regulates tissue injury and repair [19]. By utilising sophisticated analytical approaches and state-of-the-art techniques, the authors begin to describe the effect of pirfenidone in ECM production as well as innate and adaptive immunity. They also provided a roadmap to the translational study of IPF treatment, and proposed CEMIP as a target and screening marker of treatment response. This is of major interest considering the recently identified protective role of hyaluronan in post-stress fibrotic injury repair [20].

TABLE 1 Studies suggesting mechanisms of pirfenidone action

\begin{tabular}{|c|c|c|}
\hline First author [ref.] & Mechanism & Setting \\
\hline Оки [6] & Inhibition of TGF- $\beta 1, F G F, I L-1 \beta$ & In vivo (BLM model) \\
\hline ZhaNG [14] & $\begin{array}{l}\text { No effects on histopathology expression of phospho-SMAD3 and } \\
\text { senescence markers (p16, p21) }\end{array}$ & In vitro (lung tissue samples) \\
\hline KWAPISZEWSKA [15] & $\begin{array}{l}\text { Inhibition of CEMIP, TGF- } \beta 1 \\
\text { Regulation of innate and adaptive immunity }\end{array}$ & In vitro (fibroblasts, lung homogenates) \\
\hline Song [16] & Inhibition of periostin, TGF- $\beta 1$ & In vivo (BLM model) \\
\hline
\end{tabular}

TGF: transforming growth factor; FGF: fibroblast growth factor; IL: interleukin; BLM: bleomycin; CEMIP: cell migration-inducing and hyaluronan-binding protein; GLI: glioma-associated oncogene homologue. 
These novel findings must be considered in the context of the limitations of the study. The in vitro studies of pirfenidone-treated cells used a concentration of pirfenidone that is markedly higher than a level that would be achieved in patients. This raises the possibility that the findings in cultured fibroblasts may be off-target and not representative of what occurs in patients. In vivo data on the role of CEMIP in experimental models of lung fibrosis are missing. In addition, a direct mechanistic link between CEMIP and regulation of fibroblast homeostasis and hyaluronan metabolism has not been addressed. Immunolocalisation studies revealed that the effects of CEMIP on fibroblast phenotypes are not cell-specific and this begs the question of whether CEMIP inhibition in other cell types, including lung epithelial and endothelial cells, will exert similar antifibrotic effects. Finally, the expression data on lung homogenates define the net effects of pirfenidone on IPF lung tissue. They do not define whether these effects are due to direct or indirect effects of pirfenidone on the cellular and molecular pathways, or which pathways directly influence lung remodelling and fibrosis, and which are bystanders. Resolving these questions would require identifying the preferential binding partner(s) for pirfenidone.

In conclusion, this novel study applied a multilayer reductionist approach to validate key findings from global gene expression analysis, and identify novel biomarkers and possible therapeutic targets for a drug that advanced to the clinical setting with a relatively unknown mechanism of action. These findings should be of particular interest to groups developing IPF therapies that may be targeting pathways similar to those impacted by pirfenidone or that could be used synergistically in combination with pirfenidone.

Conflict of interest: A. Tzouvelekis holds a patent on inhaled or aerosolised delivery of thyroid hormone to the lung as a novel therapeutic agent in fibrotic lung diseases (OCR\#6368), disclosed to Yale University. P.J. Wolters reports receiving grants and personal fees from Genentech, and personal fees from Boehringer Ingelheim and Blade Therapeutics, outside the submitted work.

\section{References}

1 Bagnato G, Harari S. Cellular interactions in the pathogenesis of interstitial lung diseases. Eur Respir Rev 2015; 24: $102-114$

2 Wynn TA, Ramalingam TR. Mechanisms of fibrosis: therapeutic translation for fibrotic disease. Nat Med 2012; 18: $1028-1040$

3 Raghu G, Remy-Jardin M, Myers JL, et al. Diagnosis of Idiopathic Pulmonary Fibrosis. An Official ATS/ERS/JRS/ ALAT Clinical Practice Guideline. Am J Respir Crit Care Med 2018; 198: e44-e68.

4 Ryerson CJ, Kolb M. The increasing mortality of idiopathic pulmonary fibrosis: fact or fallacy? Eur Respir J 2018; 51: 1702420 .

5 Tzouvelekis A, Tzilas V, Papiris S, et al. Diagnostic and prognostic challenges in idiopathic pulmonary fibrosis: a patient "Q and A" approach. Pulm Pharmacol Ther 2017; 42: 21-24.

6 Oku H, Shimizu T, Kawabata T, et al. Antifibrotic action of pirfenidone and prednisolone: different effects on pulmonary cytokines and growth factors in bleomycin-induced murine pulmonary fibrosis. Eur J Pharmacol 2008; 590: 400-408.

7 Taniguchi H, Ebina M, Kondoh Y, et al. Pirfenidone in idiopathic pulmonary fibrosis. Eur Respir J 2010; 35: 821-829.

8 Cottin V, Koschel D, Gunther A, et al. Long-term safety of pirfenidone: results of the prospective, observational PASSPORT study. ERJ Open Res 2018; 4: 00084-2018.

9 King TE Jr, Bradford WZ, Castro-Bernardini S, et al. A phase 3 trial of pirfenidone in patients with idiopathic pulmonary fibrosis. N Engl J Med 2014; 370: 2083-2092.

10 Noble PW, Albera C, Bradford WZ, et al. Pirfenidone in patients with idiopathic pulmonary fibrosis (CAPACITY): two randomised trials. Lancet 2011; 377: 1760-1769.

11 Costabel U, Albera C, Lancaster LH, et al. An open-label study of the long-term safety of pirfenidone in patients with idiopathic pulmonary fibrosis (RECAP). Respiration 2017; 94: 408-415.

12 Ley B, Swigris J, Day BM, et al. Pirfenidone reduces respiratory-related hospitalizations in idiopathic pulmonary fibrosis. Am J Respir Crit Care Med 2017; 196: 756-761.

13 Neighbors M, Cabanski CR, Ramalingam TR, et al. Prognostic and predictive biomarkers for patients with idiopathic pulmonary fibrosis treated with pirfenidone: post-hoc assessment of the CAPACITY and ASCEND trials. Lancet Respir Med 2018; 6: 615-626.

14 Zhang Y, Jones KD, Achtar-Zadeh N, et al. Histopathologic and molecular analysis of idiopathic pulmonary fibrosis lungs from patients treated with pirfenidone or nintedanib. Histopathology 2018; in press [https://doi.org/ 10.1111/his.13745].

15 Kwapiszewska G, Gungl A, Wilhelm J. Transcriptome profiling reveals the complexity of pirfenidone effects in idiopathic pulmonary fibrosis. Eur Respir J 2018; 52: 1800564.

16 Song X, Yu W, Guo F. Pirfenidone suppresses bleomycin-induced pulmonary fibrosis and periostin expression in rats. Exp Ther Med 2018; 16: 1800-1806.

17 Kurita Y, Araya J, Minagawa S, et al. Pirfenidone inhibits myofibroblast differentiation and lung fibrosis development during insufficient mitophagy. Respir Res 2017; 18: 114

18 Didiasova M, Singh R, Wilhelm J, et al. Pirfenidone exerts antifibrotic effects through inhibition of GLI transcription factors. FASEB J 2017; 31: 1916-1928

19 Jiang D, Liang J, Noble PW. Hyaluronan in tissue injury and repair. Annu Rev Cell Dev Biol 2007; 23: 435-461.

20 Liang J, Zhang Y, Xie T, et al. Hyaluronan and TLR4 promote surfactant-protein-C-positive alveolar progenitor cell renewal and prevent severe pulmonary fibrosis in mice. Nat Med 2016; 22: 1285-1293. 